\title{
Context-Aware Mobile Media and Social Networks
}

\author{
Jonna Häkkilä \\ Nokia Research Center \\ Yrttipellontie 1 \\ 90230 Oulu, Finland \\ jonna.hakkila@nokia.com
}

\author{
Albrecht Schmidt \\ University of Duisburg-Essen \\ Schuetzenbahn 70 \\ 45117 Essen, Germany \\ albrecth.schmidt@acm.org
}

\author{
Jani Mäntyjärvi \\ VTT \\ Kaitoväylä 1 \\ 90570 Oulu, Finland \\ jani.mantyjarvi@vtt.fi
}

Alireza Sahami
University of Duisburg-Essen
Schuetzenbahn 70
45117 Essen, Germany
alireza.sahami@uni-due.de

\author{
Panu Åkerman \\ Nokia Research Center \\ Visiokatu 1 \\ 33270 Oulu, Finland
}

panu.m.akerman@nokia.com

\begin{abstract}
Context-awareness is one of the rising trends of future mobile technology, and due to advances in technology development, new application and service concepts are being developed and demonstrated in an ever-increasing manner. This workshop brings together researchers and practitioners working on humancomputer interaction (HCI) aspects of context-aware mobile technology and communities to present their insights and research on new concepts, interaction design for mobile context-awareness, usability challenges, collaborative context-aware services and applications for supporting communities, and other topics related to HCI with mobile context-aware technology.
\end{abstract}

\section{Categories and Subject Descriptors}

H.5.m [Information Interfaces and Presentation (e.g., HCI)]: Miscellaneous.

\section{General Terms}

Design, Experimentation, Human Factors.

\section{Keywords}

Context-awareness, human-computer interaction, mobile computing, social networks.

\section{INTRODUCTION}

During recent years, we have witnessed the rise of context-aware technology in research forums. Context-awareness, a state where the device is aware of the situation in which it is used, can be harnessed to serve numerous different types of use cases where the system adapts its behavior according to the circumstances. The focus of context-awareness research so far has been more on developing the enabling side of the technology including, for example, areas such as context sensing, sensor fusion, and inferring logic for context recognition. However, many of the components needed for designing and building context-aware applications and services are now in the phase that they can be utilized in practice in a mobile technology domain - for instance Copyright is held by the author/owner(s).

MobileHCI'09, September 15 - 18, 2009, Bonn, Germany.

ACM 978-1-60558-281-8. mobile phones now commonly have integrated GPS, accelerometers and other sensor functionalities. The time has now come to consider context-aware services and applications more extensively.

Mobile handheld devices, which have been highly adopted by large user groups, constitute an interesting platform for contextawareness. They are used in different kinds of dynamic situations, where the preferences of users may vary, and where different features are prioritized. While the increasing complexity and growing number of features are challenges to enabling intuitive and easy use of devices, context-awareness may enable more efficient uses of mobile applications and services e.g., by offering shortcuts to situationally-relevant information or device features. However, context-awareness also brings challenges for successful and usable UI design due to, for example, uncertainties in context recognition, difficulties in inferring the most relevant, consequent actions, and adaptive UI presentations [7]. Thus, further HCI research on context-awareness is needed. However, this problem area is already very wide, and in order to obtain a workshop with interesting, focused discussion among researchers as well as share the state-of-the art, we chose to concentrate on a narrower subtopic-context-aware mobile media and social networks.

Much of the mobile technology concentrates on communication between people, utilizing channels such as calling, SMS or MMS messaging, or mobile e-mail, making it strongly a social media. Parallel to the progress in mobile communication technologies, the Internet has matured with Web 2.0 services and social network sites, such as Facebook and MySpace, have proliferated. Taking into account today's mobile devices' capabilities in creating, sharing and showing multimedia content, we have powerful platforms to exploit context-awareness to develop new types of mobile applications that enhance social networks or provide services or information for them.

\section{RELATED WORK}

Context-awareness and its use have gained several definitions among researchers, often reflecting the technology domain it is approached from. Research on context-awareness has focused on supporting three main types of functionality: presentation of 
information and services to a user, automatic execution of a service, and tagging of context to information for later retrieval [3]. Moreover, context awareness is often categorized to pull and push [8], where the first type is initiated by the user and the second is event-triggered and initiated, for instance when deviceintegrated sensors detect a certain triggering event. In our workshop, we take the approach of Dey and Abowd to contextawareness, which broadly defines context as "any information that can be used to characterize the situation of an entity. An entity is a person, place, or object that is considered relevant to the interaction between a user and an application, including the user and applications themselves' [3]. This definition functions well in the settings of our workshop, as we are interested in MobileHCI-oriented presentations of different aspects of contextawareness without restricting the submissions to any narrow context source domain.

Context-aware mobile devices often utilize different kinds of sensors, and have been used to demonstrate various contextadaptive features. For instance, mobile device screen layout orientation, backlight, ringing tone and volume adaptation have been proposed $[5,6,9,14]$. Location-based applications form a significant area within mobile context-awareness, and have great potential for future commercial applications; this is particularly relevant with today's GPS-enabled mobile devices with mobile maps. Common applications demonstrating location-awareness are tour guides in the city, campus or museum environments, shopping assistants, messaging systems, and location-sensitive reminders $[1,2,4,13]$.

Although the most typical examples of context-awareness are still concepts that concentrate on an individual user and his/her interaction with the device, context-awareness has great potential also in facilitating social interaction and collaboration, as illustrated e.g., in [11, 12]. Osbakk and Rydgren present a presence application, where a bluetooth-triggered reminder is activated when it detects the proximity of another person's bluetooth phone [11]. Poupyrev et al. [12] have demonstrated a gadget for detecting the presence of people that are buying and selling items that are of interest to a user. Furthermore, collaborative context-enhanced communication within mobile communities provide the basis for novel media experiences [9].

\section{THE WORKSHOP}

In this workshop, we look deeper to the human-computer interaction side of mobile context-awareness for communities, focusing especially on issues of media. The workshop provides a venue to present novel research in this field and to openly discuss ideas and problems on the topic. The goal of the workshop is to share the knowledge of the current state of-the-art research in the field, chart the future challenges, and foster a peer community. This is facilitated with the presentations and discussions in the workshop.

The workshop's topics of interest include:

- Novel application and service concepts that utilize information about a community or facilitates community awareness

- Novel application and service concepts related to context-aware mobile media that involve interaction between people
- User studies on social context-aware mobile applications

- Studies on the usability and interaction design of context-aware multimedia applications

- Privacy and other social aspects of context-aware mobile applications

- Mobile application concepts that utilized distributed context-recognition

As the workshop is targeted to the MobileHCI community, the workshop presentations will highlight HCI-related perspectives rather than technical aspects. The target audience for the workshop is researchers both from academia and industry working on interaction issues related to mobile context-aware media, who are emphasizing the aspects of social interaction, collaboration and community. The design of such applications can derive contextual information from e.g., the physical environment, the user, and a community relevant for the user. The communities that are utilized can consist of various different kinds of social groups - for instance family, colleagues, currently present people, or a virtual community. In addition to novel application and service concepts, the workshop is interested in studies or designs looking at more specific HCI aspects, such as UI visualizations, interaction modalities, or interaction flows, within the workshop focus area.

The workshop home page including the accepted papers presented at the workshop can be found at [15].

\section{REFERENCES}

[1] Abowd, G. D, Atkeson, C. G., Hong, J., Long, S., Kooper R. and Pinkerton, M. 1997. Cyberguide: A Mobile ContextAware Tour Guide. ACM Wireless Networks, 3, 421-433

[2] Davies, N., Cheverst, K., Mitchell, K., and Efrat, A. 2001 Using and Determining Location in a Context-Sensitive Tour Guide. IEEE Computer 34, 8, 35-41.

[3] Dey, A.K. and Abowd, G.D. 2000. Towards a Better Understanding of Context and Context-Awareness, in: CHI 2000 Workshop on The What, Who, Where, When, Why and How of Context-Awareness, 2000

[4] Dey, A.K. and Abowd, G.D. 2000. CybreMinder: A Context Aware System for Supporting Reminders. In Proceedings of HUC, (pp. 172-186).

[5] Gellersen, H.W., Schmidt, A., Beigl, M. 2002. Multi-Sensor Context-Awareness in Mobile Devices and Smart Artefacts. Mobile Networks and Applications 7, 341-351.

[6] Hinckley, K., Pierce, J., Sinclair, M. and Horvitz, E. 2000. Sensing Techniques for Mobile Interaction. CHI Letters 2, 2 , 91-100

[7] Häkkilä, J. 2006. Usability with Context-Aware Mobile Applications - Case Studies and Design Guidelines. PhD Thesis, University of Oulu.

[8] Michahelles, F., and Samulowitz, M. 2002. Smart CAPs for SmartIts - Context Detection for Mobile Users, Personal and Ubiquitous Computing, 6, Springer-Verlag London Ltd., 2002, pp. 269-275

[9] Mäntyjärvi, J., Huuskonen, P., Himberg, J. 2002. Collaborative context determination to support mobile 
terminal applications. IEEE Wireless Communications, Oct 2002, Vol. 9 (5), pp:39- 45

[10] Mäntyjärvi, J., Tuomela, U., Känsälä, I. and Häkkilä, J. 2003. Context Studio - Tool for Personalizing Context-Aware Application in Mobile Terminals. Proc. OZCHI 2003, pp. 6473

[11] Osbakk, P., Rydgren, E. 2005. Ubiquitous Computing for the Public. In Proceedings of Pervasive 2005 Workshop on Pervasive Mobile Interaction Devices (PERMID 2005), pp. 56-59

[12] Poupyrev, P., Sasao, T., Saruwatari, S, Moridawa, H., Aoyama, T., Davis, P. Service Discovery in TinyObj: Strategies and Approaches. In Proceedings of Pervasive 2005
Workshop on Pervasive Mobile Interaction Devices (PERMID 2005), pp. 19-22.

[13] Rantanen, M, Oulasvirta, A., Blom, J., Tiitta, S., \& Mäntylä, M. 2004. InfoRadar: Group and Public Messaging in the Mobile Context. Proceedings of NordiCHI 2004, pp. 131140

[14] Schmidt, A, Gellersen, H.-W. Context-Aware Mobile Telephony. SIGGROUP Bulletin, Vol. 22, No. 1, April 2001, pp. 19-21.

[15] Workshop 'Context-Aware Mobile Media and Social Networks' web page (last accessed 20 July 2009): http://wiki.research.nokia.com/index.php/CAM3SN\#Context -Aware_Mobile_Media_and_Mobile_Social_Networks 